\title{
Gestational pulmonary arterial hypertension
}

\author{
Matthew Moll, ${ }^{1}$ Julie G. Payne, ${ }^{2}$ Melissa H. Tukey, ${ }^{2}$ Harrison W. Farber ${ }^{2}$ \\ ${ }^{1}$ Boston Medical Center, Boston, Massachusetts, USA; ${ }^{2}$ Pulmonary Center, Boston University School of Medicine, Boston, Massachusetts, USA
}

\begin{abstract}
Pulmonary arterial hypertension (PAH) is a progressive disease marked by the irreversible pulmonary vascular changes of vasoconstriction, thrombosis, and proliferation of smooth muscle and endothelial cells. The untreated clinical course is characterized by progressive dyspnea and a median survival of less than 3 years. Many of these patients are of child-bearing age; however, pregnancy leads to physiologic changes that are particularly poorly tolerated in PAH, conferring a $30 \%-56 \%$ mortality. We present a case of PAH that spontaneously resolved after termination of pregnancy and recurred during each of two subsequent pregnancies. To our knowledge, this case is unique, because no cases of spontaneous resolution of idiopathic PAH have been reported in adults, nor have there been any reports of pulmonary hypertension that is isolated to the gestational period.
\end{abstract}

Keywords: pulmonary arterial hypertension, pregnancy, gestation, pulmonary vascular disease, resolution.

Pulm Circ 2015;5(4):730-733. DOI: 10.1086/683689.

The World Health Organization (WHO) classifies pulmonary hypertension $(\mathrm{PH})$ into five groups that are based on mechanisms of disease; group I PH is defined as pulmonary arterial hypertension (PAH). ${ }^{1} \mathrm{PAH}$, characterized by vasoconstriction, thrombosis, and proliferation of smooth muscle and endothelial cells, ${ }^{2}$ is defined as a mean pulmonary arterial pressure $(\mathrm{mPAP}) \geq 25 \mathrm{mmHg}$ at rest, a mean pulmonary capillary wedge pressure or left ventricular enddiastolic pressure of $<15 \mathrm{mmHg}$, and a pulmonary vascular resistance of 3 Wood units. Among the entities included in group I PH (PAH) is idiopathic pulmonary arterial hypertension (IPAH); a rare disorder with an untreated clinical course generally characterized by progressive worsening of symptoms and cardiopulmonary hemodynamics and a median survival of less than 3 years. ${ }^{3}$ With currently available therapies, prostaglandin analogs, endothelinreceptor antagonists, phosphodiesterase inhibitors, and soluble guanylyl cyclase agonists, symptoms, hemodynamic characteristics, and survival have been improved in patients with IPAH. However, mortality is still as high as $30 \%$ over a 3-year period. ${ }^{4-6}$ Outcomes of patients who develop PAH during pregnancy are particularly poor, with an estimated maternal mortality of $30 \%-56 \%{ }^{7}$ To date, there have been no reported cases of spontaneous resolution of PAH in adults, nor have there been reports of $\mathrm{PAH}$ isolated to the gestational period. We present a unique case of PAH that was diagnosed during pregnancy, resolved after termination of pregnancy, and recurred during two subsequent pregnancies.

\section{CASE DESCRIPTION}

The patient, a 20-year-old woman who was 17 weeks pregnant (gravida 3, para 1011), presented to the emergency department (ED) unre- sponsive, febrile, hypotensive, and hypoglycemic. Initial echocardiographic examination in the ED revealed a severely dilated right ventricle (RV) with moderately depressed systolic function; initial laboratory data suggested adrenal insufficiency. The patient was admitted to the medical intensive care unit (MICU); with administration of glucocorticoids and supportive care, the patient recovered. Right heart catheterization performed after admission to the MICU demonstrated an mPAP of $38 \mathrm{mmHg}$, consistent with PH (Table 1). The patient underwent recommended termination of pregnancy. Evaluation for secondary etiologies of the $\mathrm{PH}$ included determination of thyroid-stimulating hormone levels; testing for antinuclear antibody (ANA), C3, C4, lupus anticoagulant, anticardiolipin antibodies, antineutrophil cytoplasmic antibodies (ANCA), HIV, and hepatic virus; serum and urine toxicology tests; and computerized tomographic pulmonary angiography. All had negative findings or results. Home medications included norethidrone, ibuprofen, oxycodoneacetaminophen, docusate sodium, ferrous sulfate, and prenatal vitamins. A diagnosis of IPAH was made. In addition, during this hospitalization, idiopathic Addison disease was diagnosed. Repeat right heart catheterization 4 weeks after termination of pregnancy demonstrated persistent $\mathrm{PH}$ with mPAP of $33 \mathrm{mmHg}$ (Table 1). Extensive education about the risks of pregnancy with $\mathrm{PH}$ and the need for medical therapy was provided.

However, the patient did not return for additional clinic visits and received no pulmonary vasodilator therapy. Six months after discharge from the hospital, she returned for medical attention 9 weeks pregnant. She denied any respiratory symptoms and had been taking prescribed glucocorticoid replacement therapy. Transthoracic echocardiographic examination and right heart catheterization demonstrated complete resolution of the $\mathrm{PH}$ with an $\mathrm{mPAP}$ of $19 \mathrm{mmHg}$

Address correspondence to Dr. Harrison W. Farber, Pulmonary Center, Boston University School of Medicine, Boston, MA 20118, USA. E-mail: hfarber@bu.edu.

Submitted February 3, 2015; Accepted May 13, 2015; Electronically published October 19, 2015.

(C) 2015 by the Pulmonary Vascular Research Institute. All rights reserved. 2045-8932/2015/0504-0016. \$15.00. 
Table 1. Right heart catheterization hemodynamic data during the first pregnancy, 4 weeks after termination of the first pregnancy, and during the second pregnancy

\begin{tabular}{|c|c|c|c|c|}
\hline \multirow[b]{2}{*}{ Variable } & \multicolumn{2}{|c|}{$\begin{array}{c}\text { First } \\
\text { pregnancy, by } \\
\text { weeks from } \\
\text { initial } \\
\text { presentation }\end{array}$} & \multicolumn{2}{|c|}{$\begin{array}{l}\text { Second } \\
\text { pregnancy, by } \\
\text { weeks from } \\
\text { initial } \\
\text { presentation }\end{array}$} \\
\hline & 0 & 4 & 33 & 60 \\
\hline Gestation, no. of weeks & 17 & $\ldots^{\mathrm{a}}$ & 9 & 36 \\
\hline $\mathrm{CVP}, \mathrm{mmHg}$ & 11 & 5 & 7 & 4 \\
\hline PA systolic pressure, $\mathrm{mmHg}$ & 56 & 55 & 29 & 53 \\
\hline $\begin{array}{l}\text { PA diastolic pressure, } \\
\text { mmHg }\end{array}$ & 30 & 18 & 12 & 20 \\
\hline Mean PA pressure, $\mathrm{mmHg}$ & 38 & 33 & 19 & 32 \\
\hline PCWP, mmHg & 10 & 5 & 11 & 5 \\
\hline Cardiac output, L/min & 6.5 & 4.7 & 7.6 & 5.9 \\
\hline Cardiac index, $\mathrm{L} / \mathrm{min} / \mathrm{m}^{2}$ & 3.8 & 2.6 & 4.6 & 2.8 \\
\hline $\begin{array}{l}\text { Systemic vascular resistance, } \\
\text { dynes } / \mathrm{s} / \mathrm{cm}^{5}\end{array}$ & 726 & 2,116 & 1,494 & 964 \\
\hline $\begin{array}{l}\text { Pulmonary vascular } \\
\text { resistance, dynes } / \mathrm{s} / \mathrm{cm}^{5}\end{array}$ & 332 & 520 & 138 & 325 \\
\hline
\end{tabular}

Note: Pulmonary hypertension persisted after the first pregnancy, resolved, then recurred after the first trimester of the second pregnancy. CVP: central venous pressure; PA: pulmonary artery; PCWP: pulmonary capillary wedge pressure.

${ }^{\mathrm{a}}$ Four weeks after termination of pregnancy.

(Table 2). Despite multiple discussions, the patient elected to continue the pregnancy.

At 36 weeks gestation, the patient developed dyspnea with minimal activity and lower extremity edema. Transthoracic echocardiographic examination findings now estimated the RV systolic pressure (RVSP) at $69 \mathrm{mmHg}$. Right heart catheterization confirmed recurrence of $\mathrm{PH}$ with $\mathrm{mPAP}$ of $32 \mathrm{mmHg}$, pulmonary capillary wedge pressure of $5 \mathrm{mmHg}$, and pulmonary vascular resistance of 4.1 Wood units (Fig. 1). The patient underwent emergency cesarean delivery of a healthy male child; an intrauterine device was placed for prevention of future pregnancies. After termination of the pregnancy, the symptoms and edema completely resolved.

Again, the patient did not return for clinic visits. She presented to the hospital 3 years later and was 11 weeks pregnant. She denied any respiratory symptoms; transthoracic echocardiography estimated the RVSP at $27 \mathrm{mmHg}$. The patient was again educated about the risks of pregnancy with $\mathrm{PH}$, but she again decided to continue the pregnancy. At 17 weeks gestation, surveillance echocardiogram findings estimated the RVSP at $54 \mathrm{mmHg}$ and demonstrated new dilation of the RV. The patient was asymptomatic at the time, but after extensive counseling, she opted to terminate the pregnancy. At termination, surgical sterilization was performed. She has yet to return for a follow up visit to ensure that the $\mathrm{PH}$ has resolved.

Of note, during this entire period, the patient did not receive any PAH-specific medications, diuretics, or anticoagulants. Thus, there were no confounders that could have affected the hemodynamic parameters or the clinical course of this patient.

\section{DISCUSSION}

We report, to our knowledge, the first case of spontaneous resolution of IPAH in an adult and the first case of PAH isolated to the gestational period. We document the clinical course with right heart catheterization data demonstrating existence of $\mathrm{PAH}$ after the first trimester, eventual resolution after termination of the pregnancy, and recurrence during a second gestation. We document rapidly increasing PAP by transthoracic echocardiographic examination during a third gestation; unfortunately, the patient has yet to return to medical attention after termination of that pregnancy.

\section{PAH and pregnancy}

During healthy pregnancy, blood volume increases by as much as $50 \%$, peaking at approximately 32 weeks gestation. To adapt to this increased blood volume, cardiac output increases by $30 \%-50 \%$ by the third trimester. To accommodate the increased flow and blood volume, pulmonary and systemic vascular resistances decrease; this process is mediated by progesterone and other vasodilatory agents. ${ }^{8}$ In addition, RV end-diastolic pressure increases in approximately $20 \%$ of healthy pregnancies between week 25 and week 35 of gestation, presumably because of cephalad displacement of the diaphragm and elevated intrapleural pressures. ${ }^{9}$ In women with preexisting $\mathrm{PH}$, the vasodilatory mechanisms that normally occur in pregnancy are not sufficient to alter the response of a diseased pulmonary vasculature. ${ }^{8}$ This inability to make the necessary physiologic adaptations can result in right ventricular dysfunction, failure, and even death, thus explaining the high maternal mortality rates in pregnant individuals with PAH. ${ }^{7,8}$ This can be explained, in part, by the hypercoagulability of pregnancy, thus increasing the risk of pulmonary embolism and thrombosis, hypoxemia, and pulmonary vasoconstriction. Furthermore, labor and delivery are hemodynamically stressful, because the pain and anxiety can increase heart rate and vascular resistance, and Valsalva maneuvers will decrease venous return. In the postpartum period, blood volume is increased by the autotransfusion of blood from uterine contractions and redistribution of peripheral edema to the intravascular compartment. ${ }^{10}$ For all these reasons, pregnancy is strongly discouraged in patients with PAH. Although we cannot be certain whether our patient had preexisting PAH before the initial pregnancy or whether PAH developed during that pregnancy, the development of symptoms correlated with the expected hemodynamic changes during pregnancy and resolved after termination of the first pregnancy.

\section{Potential etiologies of $\mathrm{PH}$ during pregnancy}

Patients with PAH comprise a group with common physiologic features caused by a wide range of pathologies. Given the wide range 
Table 2. Transthoracic echocardiography data recorded during the first, second, and third pregnancies

\begin{tabular}{|c|c|c|c|c|c|}
\hline \multirow[b]{2}{*}{ Variable } & \multirow{2}{*}{ 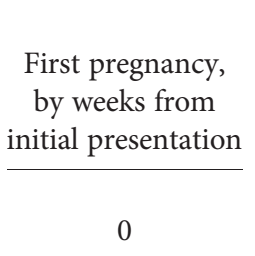 } & \multicolumn{2}{|c|}{$\begin{array}{l}\text { Second pregnancy, } \\
\text { by weeks from } \\
\text { initial } \\
\text { presentation }\end{array}$} & \multicolumn{2}{|c|}{$\begin{array}{c}\text { Third pregnancy, } \\
\text { by weeks from } \\
\text { initial } \\
\text { presentation }\end{array}$} \\
\hline & & 33 & 60 & 218 & 224 \\
\hline Gestation, no. of weeks & 17 & 9 & 36 & 11 & 17 \\
\hline Estimated RA pressure, $\mathrm{mmHg}$ & 15 & 3 & 8 & 3 & 3 \\
\hline Estimated RVSP, mmHg & 69 & 19 & 69 & 27 & 54 \\
\hline Left ventricular ejection fraction, $\%$ & 65 & 70 & 65 & 60 & 65 \\
\hline Tricuspid regurgitation severity grade & $2+$ & Trace & $2+$ & $1+$ & $1+$ \\
\hline
\end{tabular}

Note: The estimated right ventricular systolic pressure (RVSP) correlated with right heart catheterization data during the first and second pregnancies. The echocardiography data demonstrate elevated pulmonary artery pressures after the first trimester during three pregnancies. The patient did not return for right heart catheterization in her third pregnancy. RA: right atrial; RVSP: right ventricular systolic pressure.

of underlying causes, it is likely that the development of $\mathrm{PAH}$ is multifactorial. The multiple-hit hypothesis of $\mathrm{PAH}$ pathogenesis proposes that factors involving genetic background, modifier genes, and environmental triggers lead to $\mathrm{PH}^{2}$ In support of this hypothesis, mutations in the bone morphogenic protein receptor type 2 have been identified in hereditary PAH. ${ }^{11,12}$ Furthermore, several systemic conditions predispose to the development of $\mathrm{PH}$ (associated $\mathrm{PAH}){ }^{2}$ In the current patient, there was no family history of $\mathrm{PH}$ nor were there any underlying entities identified that increased her risk of $\mathrm{PH}$ or $\mathrm{PAH}$.

However, our patient does appear to possess a predisposition for $\mathrm{PAH}$, with pregnancy triggering the development of reversible PAH. It is difficult to define the underlying genetic background and modifier genes relevant to the development of PAH in this patient; however, we hypothesize that she may have had diminished production of or response to the vasodilatory mediators that decrease pulmonary vascular resistance during pregnancy.

Secondary causes of $\mathrm{PH}$ were extensively investigated, but none were found. Although thromboembolism is common in pregnancy, computerized tomographic pulmonary angiography revealed no evidence of pulmonary emboli. Moreover, it is unlikely that acute or chronic pulmonary emboli would have regressed without anticoagulant treatment. It is also possible that the patient had an autoimmune disease that was activated by pregnancy, but ANA tests, thyroid studies, ANCA tests, complement levels, HIV tests, and antiphospholipid antibody tests had negative results. $\mathrm{PH}$ has been described in POEMS (polyneuropathy, organomegaly, endocrinopathy, monoclonal protein, and skin abnormalities) syndrome, but the patient did not have the peripheral neuropathy required for this diagnosis. Therefore, if there was an underlying autoimmune process, it may be an autoimmune disorder that is poorly defined or lacks a readily available high-sensitivity assay. In addition, the Addison disease was treated and well-controlled during the subsequent episodes of PAH.

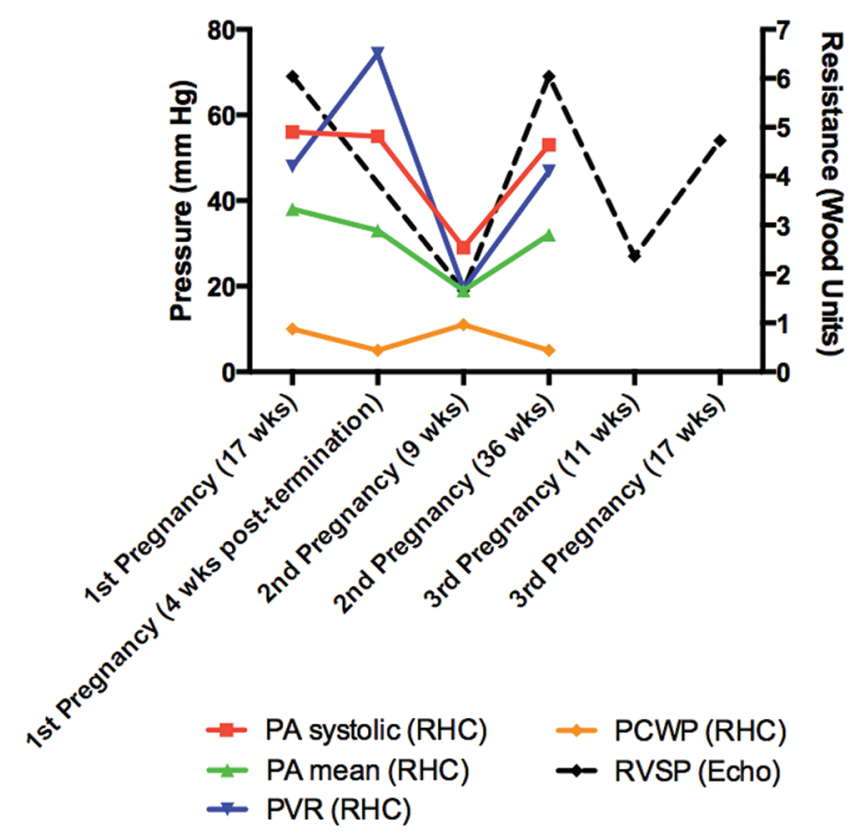

Figure 1. Pulmonary hemodynamic characteristics measured by right heart catheterization (RHC) and transthoracic echocardiography show the development of elevated pulmonary artery (PA) pressures after the first trimester during three pregnancies, persistence of pulmonary hypertension after the first pregnancy, and resolution of pulmonary hypertension during the first trimester of the second and third pregnancies. The pulmonary capillary wedge pressure (PCWP) obtained by RHC confirms the absence of left heart failure (orange line), and PA pressures are estimated by transthoracic echocardiography during the third pregnancy (black dashed line). The left vertical axis denotes pressure; the right vertical axis denotes pulmonary vascular resistance (PVR) in Wood units. Echo: transthoracic echocardiography; RVSP: right ventricular systolic pressure. 
Finally, to our knowledge, there are no reported cases of PAH associated with adrenal insufficiency (Addison disease). There is a case report of $\mathrm{PH}$ and adrenal insufficiency during pregnancy due to antiphospholipid syndrome (APS). However, that patient met the Sydney criteria for primary APS, had empty sella on brain imaging, biochemical evidence of panhypopituitarism, and positive anticardiolipin antibodies. ${ }^{13}$ Our patient did not have empty sella on brain imaging, had normal production of other pituitary hormones, and had no biochemical or clinical evidence to support a diagnosis of APS.

\section{PAH prognosis}

Mortality in patients with IPAH is mainly due to right ventricular dysfunction and failure. Currently available medical therapies have improved clinical status, morbidity, and mortality in patients with IPAH, but mortality is still extremely high..$^{4-6}$ In 1991, the National Institutes of Health Registry reported a survival of $47 \%$ during a 3 -year period. ${ }^{3}$ More recently, the Registry to Evaluate Early and Long-Term PAH Disease Management (REVEAL) ${ }^{5}$ and French registries ${ }^{6}$ have reported a survival rate of $67 \%$. In this context, the case presented is significant because it is the first report of spontaneous resolution of WHO group I PAH in an adult. Interestingly, there have been two case reports of IPAH that developed in adolescence and resolved in adulthood. ${ }^{14,15}$ In each of these case reports, individuals developed exercise intolerance and $\mathrm{PH}$ around 10 years of age. By 20 years of age, both patients had marked improvement in their symptoms without treatment; in each case, resolution of $\mathrm{PH}$ was demonstrated by right heart catheterization. The occurrence of reversible PAH reported during adolescence or pregnancy may suggest that hormones and growth factors contribute to changes in pulmonary vascular resistance. Identification and further investigation of patients with gestational PAH may be important for both patient care and for defining a research model to understand any potential reversible aspects of $\mathrm{PAH}$ pathogenesis.

\section{Conclusions}

In conclusion, we report the first case of PAH seemingly only apparent during gestation and with complete resolution after termination of pregnancy. It is difficult to determine the pathogenesis of the $\mathrm{PAH}$ in this patient, but it may be due to decreased production of or response to vasodilatory mediators in the pulmonary vasculature. Gestational PAH may occur more frequently than recognized, because there are multiple other reasons for dyspnea in this population. However, if no other obvious reason for dyspnea is found in a pregnant patient, it should be considered. Identifying this entity may be important for counseling patients about prognosis and fu- ture pregnancies. Finally, the reversibility of the PAH in this patient is notable, because it is the first case of spontaneous resolution of IPAH in adults. Therefore, such patients may provide a model in which any reversible aspects of IPAH could be investigated.

\section{Source of Support: Nil.}

Conflict of Interest: None declared.

\section{REFERENCES}

1. Simonneau G, Gatzoulis, MA, Adatia, I, Celemajer D, Denton C, Ghofrani A, Gomez Sanchez MA, et al. Updated clinical classification of pulmonary hypertension. J Am Coll Cardiol 2013;62:D100-D108.

2. Farber HW, Loscalzo J. Pulmonary arterial hypertension. N Engl J Med 2004;351:1655-1665.

3. D'Alonzo GE, Barst RJ, Ayres SM, Bergofsky EH, Brundage BH, Detre KM, Fishman AP, et al. Survival in patients with primary pulmonary hypertension: results from a national prospective registry. Ann Intern Med 1991;115:343-349.

4. Zamanian RT, Kudelko KT, Sung YK, de Jesus Perez V, Liu J, Spiekerkoetter E. Current clinical management of pulmonary arterial hypertension. Circ Res 2014;115:131-147.

5. Benza RL, Miller DP, Barst RJ, Badesch DB, Frost AE, McGoon MD. An evaluation of long-term survival from time of diagnosis in pulmonary arterial hypertension from the REVEAL Registry. Chest 2012;142:448556.

6. Humbert M, Sitbon O, Yaïci A, Montani D, O'Callahan DS, Jaïs X, Parent F, et al; French Pulmonary Arterial Hypertension Network. Survival in incident and prevalent cohorts of patients with pulmonary arterial hypertension. Eur Respir J 2010;36:549-555.

7. Weiss BM, Zemp L, Seifert B, Hess OM. Outcome of pulmonary vascular disease in pregnancy: a systematic overview from 1978 through 1996. J Am Coll Cardiol 1998;31:1650-1657.

8. Obican SG, Cleary KL. Pulmonary arterial hypertension in pregnancy. Semin Perinatol 2014;5:289-294.

9. Bader RA, Bader ME, Rose DJ, Braunwald E. Hemodynamics at rest and during exercise in normal pregnancy as studied by cardiac catheterization. J Clin Invest 1955;34:1524-1536.

10. Gei A, Montufar-Rueda C. Pulmonary hypertension and pregnancy: an overview. Clin Obstet Gynecol 2014;57:806-826.

11. Nichols WC, Koller DL, Slovis B, Foroud T, Terry VH, Arnold ND, Siemieniak DR, et al. Localization of the gene for familial primary pulmonary hypertension to chromosome 2q31-32. Nat Genet 1997;15:277280.

12. Morse JH, Jones AC, Barst RJ, Hodge SE, Wilhelmsen KC, Nygaard TG. Mapping of familial primary pulmonary hypertension locus (PPH1) to chromosome 2q31-32. Circulation 1997;95:2603-2606.

13. Alfadhli EM. Secondary adrenal insufficiency and primary antiphospholipid syndrome. Inter Med 2009;48:1963-1966.

14. Fujii A, Rabinovitch M, Matthew EC. A case of spontaneous resolution of idiopathic pulmonary hypertension. Br Heart J 1981;46:574577.

15. Bourdillon PDV, Oakley CM. Regression of primary pulmonary hypertension. Br Heart J 1976;38:264-270. 